\title{
Diagnóstico y tratamiento de coledocolitiasis con diferentes técnicas de imagen
}

\author{
Diagnosis and treatment of choledocholithiasis with different \\ imaging techniques
}

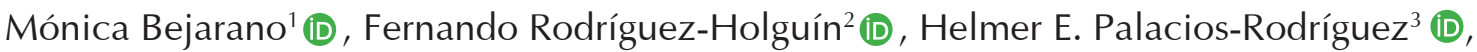
Christian E. Delgado-Ocampo ${ }^{3}$

1 MD., MSc., MACC, especialista en Cirugía general, magister en Epidemiología, Departamento de Cirugía General, Fundación Valle del Lili, Cali, Colombia.

2 Médico, especialista en Cirugía general y Cirugía de Trauma y Emergencias, Departamento de Cirugía de Trauma y Emergencias, Fundación Valle del Lili, Cali, Colombia.

3 Médico, especialista en Cirugía general, Fellowship de Cirugía de Trauma y Emergencias, Universidad del Valle, Cali, Colombia.
\end{abstract}

\section{Introducción}

En pacientes con sospecha de coledocolitiasis, el estudio mediante colangiografía, endoscópica (CPRE), por resonancia magnética (CRM) o intraoperatoria (CIO), permite definir la necesidad de la exploración de la vía biliar ${ }^{1}$, bien sea quirúrgica, endoscópica o percutánea. El estudio multicéntrico del Congreso Quirúrgico de Suroeste, con 1263 pacientes, mostró un desempeño diagnóstico similar entre estas tres opciones, sin diferencias estadísticamente significativas ${ }^{1}$.

En nuestro medio, cuando hay sospecha de coledocolitiasis, se prefiere la CRM al no ser invasiva, y por ende, tener menos riesgos de complicaciones, aunque presenta ciertas limitaciones, como una baja resolución espacial, con una sensibilidad de $64 \%$ para cálculos menores de $3 \mathrm{~mm}$, y ser susceptible de errores por artefactos de movimiento, por lo que baja su rendimiento en pacientes que no toleren permanecer inmóviles durante el tiempo que requiere una resonancia ${ }^{2,3}$.

\section{Presentación del caso}

Paciente masculino de 83 años, con antecedentes de hipertensión arterial, gastritis crónica, dislipidemia y colelitiasis, con episodios recurrentes de cólico biliar y coluria, quien consulta por 4 días de

Palabras clave: colelitiasis; conducto colédoco; coledocolitiasis; colangiografía; imagen por resonancia magnética; colangiopancreatografia retrógrada endoscópica.

Keywords: cholelithiasis; common bile duct; choledocholithiasis; cholangiography; magnetic resonance imaging; endoscopic retrograde cholangiopancreatography.

Fecha de recibido: 30/06/2021 - Fecha de aceptación: 07/07/2021 - Publicación en línea: 17/09/2021

Autor de correspondencia: Mónica Bejarano, Carrera 59 \#11B-56, Cali, Colombia. Teléfono: 315-5574039

Dirección electrónica: monicirugia@gmail.com

Citar como: Bejarano M, Rodríguez-Holguín F, Palacios-Rodríguez HE, Delgado-Ocampo CE. Diagnóstico y tratamiento de coledocolitiasis con diferentes técnicas de imagen. Rev Colomb Cir. 2022;37:135-8. https://doi.org/10.30944/20117582.985

Este es un artículo de acceso abierto bajo una Licencia Creative Commons - BY-NC-ND https://creativecommons.org/licenses/by-nc-nd/4.0/deed.es 
dolor y distensión abdominal, un episodio emético y ausencia de deposiciones, con flatos presentes. Al ingreso normotenso, afebril, anictérico, con abdomen distendido, sin masas ni signos de irritación peritoneal. Los paraclínicos mostraron patrón colestásico, con bilirrubina total de 2,96 $\mathrm{mg} / \mathrm{dl}$, bilirrubina directa de 2,06 mg/dl, fosfatasa alcalina de $260 \mathrm{UI} / \mathrm{L}$, gamma glutamil transpeptidasa de $403 \mathrm{U} / \mathrm{l}$, alanino aminotransferasa (ALT) de $114 \mathrm{U} / \mathrm{l}$ y aspartato aminotransferasa (AST) de 135,5 U/l, sin leucocitosis ni neutrofilia.

Se realizó ecografía hepatobiliar que reportó ventana limitada, observando signos no claros de colelitiasis, sin poder caracterizar adecuadamente la pared vesicular, y sin alteraciones en la vía biliar, con colédoco de 2,8 $\mathrm{mm}$. En vista de esto,

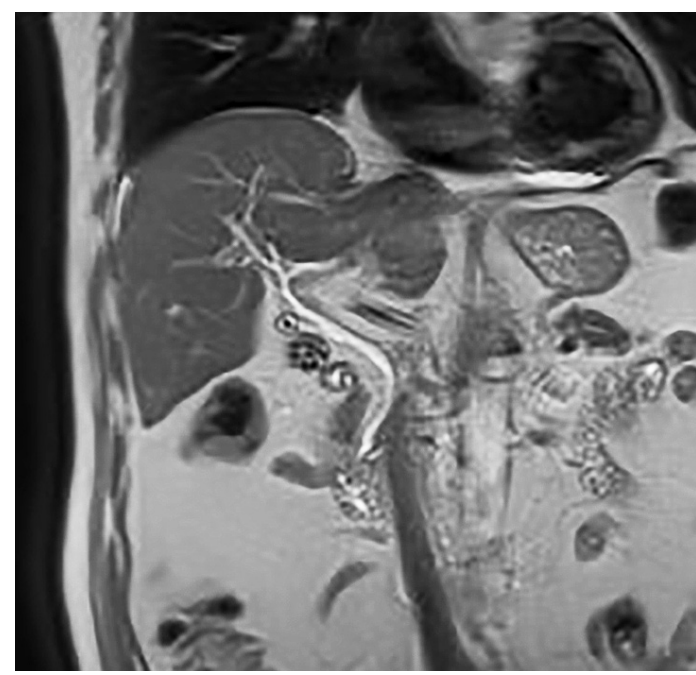

Figura 1. Colangiografía por resonancia magnética donde no se aprecian cálculos en la via biliar, dilatación ni estenosis. se realizó CRM donde informaron colelitiasis sin signos de coledocolitiasis, con vía biliar intra y extrahepática de curso, calibre y ramificación normal, con artificios por movimiento que limitan la calidad del estudio (Figura 1).

Con estos datos, se llevó el paciente a cirugía para colecistectomía laparoscópica y colangiografía intraoperatoria, en la que se identificó coledocolitiasis múltiple (Figura 2). Se practicó la exploración de vía biliar laparoscópica y utilizando un catéter Fogarty $3 \mathrm{~F}$ se extrajeron siete cálculos de la vía biliar a través del cístico (Figura 3). La CIO de control no mostró cálculos residuales, tras lo cual se completó la colecistectomía. El paciente evolucionó adecuadamente, con normalización de pruebas hepáticas y se egresa al segundo día postoperatorio.

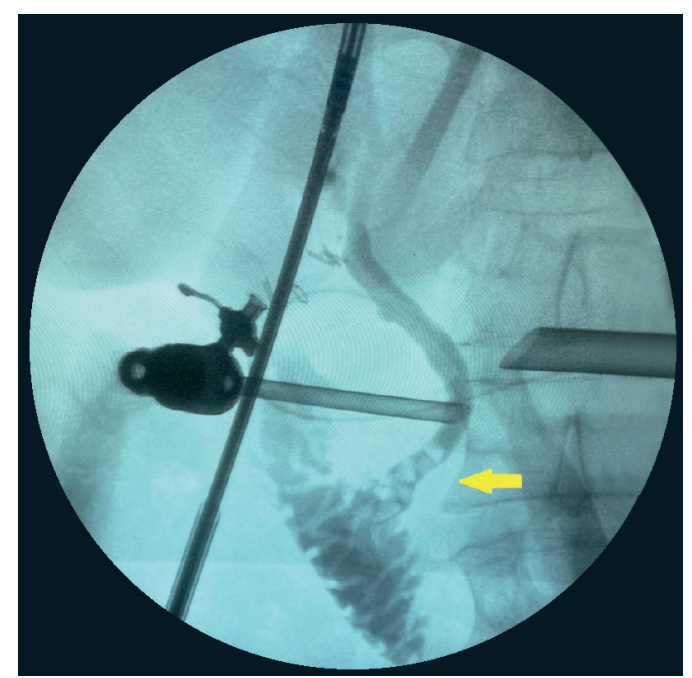

Figura 2. Colangiografia intraoperatoria donde se aprecian varios defectos de llenamiento (flecha) correspondientes a calculos.

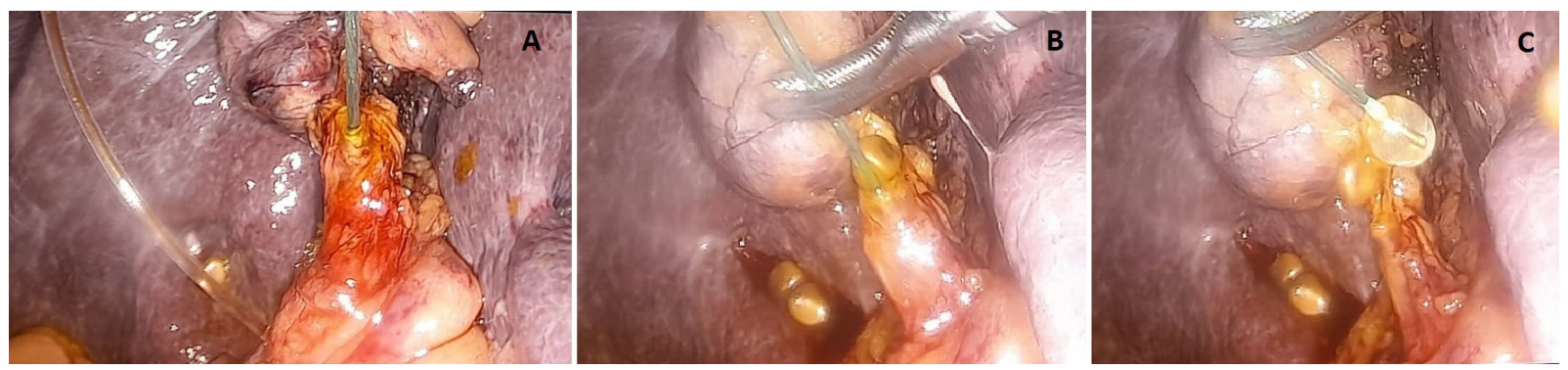

Figura 3. Extraccion transcistica de calculos en la via biliar utilizando un cateter Fogarty. 


\section{Discusión}

La colelitiasis es una patología muy común en la población occidental, con una incidencia estimada del $10-15 \%{ }^{4}$, y la colecistectomía por laparoscopia es el manejo estándar. La presencia o la sospecha de coledocolitiasis obligan al cirujano a escoger entre diversas opciones para resolverla.

Actualmente se dispone de diversos métodos para el estudio de las patologías de la vía biliar, unos diagnósticos no invasivos y otros invasivos con fines diagnósticos y terapéuticos. Estudios prospectivos, como el de Silva y colaboradores ${ }^{5}$, que comparó el desempeño de CPRE y CRM en 284 pacientes, mostraron resultados similares al estudio multicéntrico del Congreso Quirúrgico de Suroeste ${ }^{1}$, con sensibilidad del $98,3 \%$ y especificidad del 89,7 \% para la CRM, con la ventaja de ser menos operador dependiente. Para otros autores, la CRM se ha convertido en el estándar de oro para el estudio de las vías biliares, por su alta precisión en la evaluación de cálculos ${ }^{6,7}$.

El paciente de este caso contaba con predictores de coledocolitiasis por clínica (coluria) y exámenes de laboratorio (elevación de enzimas hepáticas con patrón obstructivo), sin embargo, en las dos imágenes diagnósticas iniciales no se observó dilatación de la vía biliar o calculo en la vía biliar. Por esa razón, se decidió utilizar uno de los métodos invasivos, diagnóstico y terapéutico, como la CIO por vía laparoscópica, consiguiendo resolver la causa de la obstrucción de la vía biliar, con un desenlace satisfactorio.

Con este caso queremos mostrar mediante imágenes las limitaciones de la CRM. En pacientes con sospecha de coledocolitiasis y CRM negativa, en quienes no se justifique la invasión endoscópica, una anestesia y estadía intrahospitalaria adicional que significaría realizar una CPRE, la CIO por vía laparoscópica es una opción adecuada, con un desempeño diagnóstico y terapéutico similar, ideal para los pacientes en quienes se requiere realizar también una colecistectomía ${ }^{7,8}$.

\section{Cumplimiento de normas éticas}

Consentimiento informado: Este estudio es una revisión de historia clínica retrospectiva y las imágenes no permiten la identificación del paciente, por lo que no hay necesidad de un consentimiento informado.

Declaración de conflicto de intereses: Los autores declaran que no tienen conflicto de intereses.

Fuentes de financiación: Recursos propios de los autores.

\section{Contribución de los autores}

- Concepción y diseño del estudio: Monica Bejarano, Fernando Rodríguez-Holguín.

- Adquisición de datos: Monica Bejarano, Helmer E. Palacios-Rodríguez, Christian E. Delgado-Ocampo.

- Análisis e interpretación de datos: Monica Bejarano, Helmer E. Palacios-Rodríguez, Christian E. DelgadoOcampo.

- Redacción del manuscrito: Monica Bejarano, Helmer E. Palacios-Rodríguez, Christian E. Delgado-Ocampo.

- Revisión crítica: Monica Bejarano, Fernando RodríguezHolguín, Helmer E. Palacios-Rodríguez, Christian E. Delgado-Ocampo.

\section{Referencias}

1. Frazee R, Regner J, Truitt MS, Agrawal V, Swope M, Burlew CC, et al. The southwestern surgical congress multi-center trial on suspected common duct stones. Am J Surg. 2019;217:1006-9.

https://doi.org/10.1016/j.amjsurg.2018.12.062

2. Onofre-Castillo J. Sensibilidad y especificidad de colangiorresonancia en el diagnóstico de obstrucción de la vía biliar. An Radiol México. 2010;9:123-9.

3. Leal CA, Ortega DJ, Pedraza M, Cabrera LF, Sánchez S. Eficacia de la colangiopancreatografía por resonancia magnética para el diagnóstico de coledocolitiasis de probabilidad intermedia. Rev Colomb Cir. 2019;37-44. https://doi.org/10.30944/20117582.96

4. Iranmanesh P, Frossard JL, Mugnier-Konrad B, Morel P, Majno P, Nguyen-Tang T, et al. Initial cholecystectomy vs sequential common duct endoscopic assessment and subsequent cholecystectomy for suspected gallstone migration: a randomized clinical trial. JAMA. 2014;312:137-44. https://doi.org/10.1001/jama.2014.7587

5. Armando U, Martínez DL. Colangiorresonancia magnética versus hallazgos en colangiopancreatografía retrógrada endoscópica en pacientes con coledocolitiasis. Experiencia en el Hospital Regional "Gral. Ignacio Zaragoza”, ISSSTE. An Radiol México. 2011;10:98-105. 
6. Arrivé L, Hodoul M, Arbache A, Slavikova-Boucher L, Menu Y, El Mouhadi S. Magnetic resonance cholangiography: Current and future perspectives. Clinics and Research in Hepatology and Gastroenterology. 2015;39:659-64.

http://dx.doi.org/10.1016/j.clinre.2015.07.005

7. Perales SR, Souza LR, Crema E. Comparative evaluation of magnetic resonance cholangiopancreatography and perioperative cholangiography in patients with suspect choledocholithiasis. ABCD Arq Bras Cir Dig. 2019;32:e1416.

https://doi.org/10.1590/0102-672020180001e1416

8. Hall C, Regner JL, Schroeppel T, Rodriguez J, McIntyre R, Wright F, et al. Protocol driven management of suspected common duct stones: A Southwestern Surgical Congress multi-centered trial. Am J Surg. 2019;218:1152-5. https://doi.org/10.1016/j.amjsurg.2019.09.009 